\title{
Atrial natriuretic peptide and posterior pituitary neurohormone changes in patients with acute schizophrenia
}

This article was published in the following Dove Press journal:

Neuropsychiatric Disease and Treatment

\author{
Derya Guzel' \\ Ahmet Bulent Yazici² \\ Tugba Mutu Pek ${ }^{2}$ \\ Songul Doganay' \\ Alime Burcin Saykan \\ Simsek ${ }^{2}$ \\ Kadir Saglam' \\ Caglar Turan² \\ Esra Yazici ${ }^{2}$ \\ 'Department of Physiology, School of \\ Medicine, Sakarya University, Sakarya, \\ Turkey; ${ }^{2}$ Department of Psychiatry, \\ School of Medicine, Sakarya \\ University, Sakarya, Turkey
}

Correspondence: Ahmet Bulent Yazici Department of Psychiatry, Sakarya University, Medical Faculty, Korucuk Street, Sakarya 54290, Turkey

Tel +905325994988

Fax +90264255 2105

Email a.bulentyaz@gmail.com
Objectives: Interactions between neuropeptides and psychiatric disorders have been investigated for many years. The aim of this study was to evaluate oxytocin (OXT), arginine-vasopressin (AVP), and atrial natriuretic peptide (ANP) and assess their interactions with each other, as well as investigate these changes with the manifestations of schizophrenia.

Participants and methods: Thirty-four individuals having acute schizophrenia and 24 healthy individuals as the control group were included in the study. Positive and Negative Syndrome Scales, Global Assessment of Functionality score, and Clinical Global Impression (CGI) scores were measured. Serum hormone levels were analyzed using enzyme-linked immunosorbent assay and were compared with the clinical findings.

Results: OXT levels were significantly lower and AVP levels were significantly higher in patients having acute schizophrenia than the control group. OXT was negatively correlated with Positive and Negative Syndrome Scales positive score and CGI score, while it was positively correlated with Global Assessment of Functionality score. AVP was negatively correlated with CGI score. ANP levels of the patients having schizophrenia were lower than the control group; however, there was no significant correlation with clinical findings.

Conclusion: The obtained data indicate that the AVP level was higher, but OXT and ANP levels were lower in the patients having acute schizophrenia. Specifically OXT is related with reduced disease severity and increased functionality.

Keywords: schizophrenia, oxytocin, PANS, GAF, CGI, AVP

\section{Introduction}

Schizophrenia is a severe mental disorder involving delusions of thought, perception, attention, affect, and behavior, as well as heavy losses in interpersonal relationships, work life, and collective harmony. ${ }^{1}$ Schizophrenia effects almost $1 \%$ of the population worldwide and usually begins during youth. Although there is no significant difference in the frequency and prevalence of this disease between males and females, it has been shown in recent studies that males have this disease more commonly. ${ }^{2}$ DSM 5 defines an acute episode for a schizophrenia patient as one who has active symptoms for at least 1 month, without treatment (or $<1$ month with treatment). The etiology of schizophrenia may be due to one of the following: stress-prone, environmental and psychosocial factors, infectious and inflammatory factors, and neurohormonal interactions. ${ }^{3-5}$ Hypothalamic-pituitary-adrenal axis (HPA) plays the major role for the neurohormonal interactions in schizophrenia. ${ }^{6}$ Oxytocin (OXT), composed of 9 amino acids, is produced by paraventricular and supraoptic nucleus of the hypothalamus and released by the posterior pituitary gland. OXT acts as a neurotransmitter in the brain and 
regulates social interactions and sexual reproduction, playing a role in maternal-infant bonding primarily. Besides these general features, animal studies of schizophrenia models have demonstrated that OXT improves emotional discrimination and reverses social behaviors. ${ }^{7,8}$ OXT also protects the hippocampus from harmful effects of stress hormones, suppressing stress-induced cortisol release, reducing anxiety, and creating a sense of confidence in the HPA axis. ${ }^{9}$

Arginine-vasopressin (AVP) is produced by supraoptic and paraventricular nucleus and released by the posterior pituitary gland. AVP is known for its important role in body fluid balance; however, the evidence is increasingly pointing out that it has an important role in the brain as a neurotransmitter and regulates the functions of corticotropic cells in the pituitary. ${ }^{10,11}$ Atrial natriuretic peptide (ANP) is another peptide hormone which has strong natriuretic, diuretic, and vasoactive properties and is synthesized in the muscle cells of the heart atrium and released into the plasma. ${ }^{12}$ ANP receptors are found in the kidney glomeruli, mesangial cells, and central nervous system (bulbus, lateral tractus, nucleus accumbens, dorsomedial, suprachiasmatic hypothalamic nucleus, etc) in varying concentrations. In addition, ANP is produced in the brain, and it moderates OXT release. ${ }^{13}$ ANP also acts as an inhibitory regulating hormone in the HPA system. ${ }^{11}$ There are limited data about the alterations of these neurohormones in schizophrenia. The aim of our study was to evaluate neurohormones and their interactions with each other, and their relationship with the clinical features of the disease.

\section{Participants and methods Study design}

The study was designed as a cross-sectional study at the psychiatry inpatient clinic of our Medical Faculty Hospital. Patients who were hospitalized with acute schizophrenia diagnosis were included in the study. These patients were assessed at the first week of their hospitalization. Ethics committee approval was received from the Sakarya University Medical Faculty ethics committee. Patients were interviewed by a psychiatrist and were evaluated according to the criteria of DSM-V. The sample was formed as 2 groups: schizophrenia patients with acute episode $(n=34)$ and healthy individuals, who voluntarily participated in the study $(n=24)$. All participants or legal guardian provided written informed consent. The schizophrenia and control groups were only selected among the male patients with the aim of avoiding the confounding effect of gender. Body mass index (BMI), educational status, duration of the disease, the drugs used, and demographic data were recorded for each patient. All patients received drug treatment in the usual order, according to the prevalent standards for acute schizophrenia.

\section{Assessment measures}

Positive and negative syndrome scale (PANSS)

The PANSS, which was developed by Kay et al, ${ }^{14}$ is a semistructured interview scale including 30 items rating the severity with a 7-point severity rating. Eighteen of these 30 items were adapted from the "Brief Psychiatric Rating Scale" and 12 were adapted from the "Psychopathology Assessment Scale." Among 30 psychiatric parameters assessed by PANSS, 7 items belong to the positive syndrome subscale, 7 to the negative syndrome subscale, and the remaining 16 items belong to the general psychopathology subscale. All items have been identified in PANSS, and detailed explanations accompany each of the 7 severity points of these items. The increased levels of psychopathology are reflected in the 7 -point evaluation. These are: $1=$ no, $2=$ very light, $3=$ light, $4=$ moderate, $5=$ moderate/heavy, $6=$ heavy, and $7=$ very heavy. PANSS scoring is carried out by adding up the scores for each item. The potential score range for positive and negative syndrome subscales is 7-49, and for general psychopathology subscale, it is in the range of 16-112. PANSS assessments are usually performed by taking a defined period of time including the most recent week into account. The information is collected both by clinical interviews and through consultation with family members or, if the patient is in hospital, with the health professionals that are primarily responsible of the patient. PANSS evaluation is performed following a 30-40 minute semistructured interview. ${ }^{14}$ The validity and reliability study of the scale for Turkish was conducted by Kostakoğlu et al. ${ }^{15}$

\section{Clinical global impression scale (CGI)}

CGI was developed by Guy ${ }^{16}$ to assess the clinical course of all psychiatric disorders of all ages. CGI is a 3-dimensional ("severity of the disorder," "healing," and "severity of side effects") scale and is filled during a semistructured interview to assess the responses of people with psychiatric disorders to the treatments. In this study, only the subscale of "severity of the disorder" was assessed. ${ }^{16}$

\section{Global assessment of functionality (GAF)}

In its general context, GAF is a scale that is used to track the clinical course of the people through a single measure. While only the psychological, social, and occupational functioning is rated using the GAF scale, functional impairments due to 
physical or environmental constraints cannot be assessed separately. The evaluation performed using this scale is done by grading the functionality of a person by assigning a score in the range of $1-100$ by the clinician for the current period or for a period in the past. ${ }^{17}$

\section{Laboratory analysis}

At the first day of their hospitalization, blood samples from the patients and the control group were taken into tubes without anticoagulant (BD Vacutainer K2 plus plastic tubes, Becton Dickinson, Franklin Lakes, NJ, USA), and these samples were allowed to clot completely at room temperature; then, the sera were separated by centrifuging them at $4^{\circ} \mathrm{C}$ at $5,000 \times g$ for 5 minutes, and they were stored at $-80^{\circ} \mathrm{C}$ until the day of biochemical analyses.

To determine the serum OXT, AVP, and ANP levels, commercial kits were used following the kit protocols, and solid-phase sandwich enzyme-linked immunosorbent assay (ELISA) method (BioTek ELX50 Reader, BioTek, Instruments, Winooski, VT, USA and BioTek ELX-800 Washer, BioTek Instruments) was applied. OXT (Elabscience ELISA, Catalog No: E-EL-0029, Houston, TX, USA) standards with known concentrations and serum samples were placed into ELISA plates coated with human OXT-specific monoclonal antibodies. Following the incubation, elimination, and color reaction phases, a color change was observed, and it was evaluated by measuring and expressed in $\mathrm{pg} / \mathrm{mL}$. Similar procedures were applied for serum AVP level (Elabscience ELISA, Catalog No: E-EL-H0272), and serum ANP levels (Elabscience ELISA, Catalog No: E-EL-H0532). All biomarker samples were read on the ELISA reader (BioTek, Epoch) at $450 \mathrm{~nm}$ wavelength. The results were expressed in $\mathrm{pg} / \mathrm{mL}$, taking the given sensitivity values into consideration and calculating from the standard curve.

\section{Statistical analysis}

SPSS for Windows 22.0 software package (IBM Corporation, Armonk, NY, USA) was used in the statistical evaluation of the data in the study. Student's $t$-test was used to compare the mean of the variables that fit the normal distribution, and Mann-Whitney $U$ test was used for the variables that did not fit the normal distribution in the comparison of the categorical variables. Shapiro-Wilk test used for evaluating the normality of distribution of the variables. Correlations of linear variables were evaluated by Pearson correlation analysis. Spearman's correlation analysis was used for the variables that did not fit the normal distribution. Significance level was accepted as $P<0.05$.

\section{Results \\ General characteristics and clinical features of patients}

Thirty-four male acute schizophrenia patients and 24 male healthy subjects were involved in the study. There was no statistically significant difference between groups in terms of age (schizophrenia group's mean age was $38.9 \pm 13.57$ years, and control group's mean age was $40.75 \pm 10.28$ years) and BMI (schizophrenia group's mean BMI was 26.22 \pm 5.79 and control group's mean BMI was 26.78 \pm 4.11 ). Clinical and demographic properties of acute schizophrenia patients are presented in Table 1.

\section{Comparisons of the biochemical parameters}

OXT levels were significantly lower and AVP levels were significantly higher in patients with acute schizophrenia than the individuals in the control group. ANP levels were not significantly different (Table 2).

\section{Correlation of hormonal levels and clinical features in patients with acute schizophrenia}

OXT was negatively correlated with PANSS positive score and CGI score while it was positively correlated with GAF score. AVP was negatively correlated with CGI score. There was no correlation with other parameters. Correlation of biochemical parameters with all clinical features of patients is summarized in Table 3 and Figure 1.

\section{Discussion}

The main findings of the present study are about posterior pituitary neurohormones and ANP changes in patients with

Table I General characteristics and clinical features of patients

\begin{tabular}{ll}
\hline Clinical features of patients & Mean \pm SD \\
\hline Age & $38.9 \pm 13.5$ \\
Duration of disease & $163.6 \pm 151.9$ \\
Age of the onset of schizophrenia & $25.3 \pm 9.1$ \\
Number of hospitalizations & $4.1 \pm 3.0$ \\
Total duration of hospitalizations & $85.8 \pm 125.2$ days \\
PANSS positive score & $26.0 \pm 7.2$ \\
PANSS negative score & $18.2 \pm 9.3$ \\
PANSS general score & $37.7 \pm 7.8$ \\
Total PANSS score & $81.17 \pm 19.34$ \\
GAF score & $33.1 \pm 11.4$ \\
CGI-S score & $5.8 \pm 1.5$ \\
\hline
\end{tabular}

Abbreviations: CGI-S, Clinical Global Impression-Severity; GAF, Global Assessment of Functionality; PANSS, Positive and Negative Syndrome Scale. 
Table 2 Comparisons of the biochemical parameters of both groups

\begin{tabular}{|c|c|c|c|}
\hline $\begin{array}{l}\text { Biochemical } \\
\text { parameters }\end{array}$ & $\begin{array}{l}\text { Acute schizophrenia, } \\
\mathrm{n}=\mathbf{3 4} \text {, mean } \pm \text { SD }\end{array}$ & $\begin{array}{l}\text { Control } \\
\text { group, } n=24, \\
\text { mean } \pm \text { SD }\end{array}$ & $P$-values \\
\hline ANP $(\mathrm{pg} / \mathrm{mL})$ & $218.24 \pm 89.13$ & $243.0 \pm 56.61$ & 0.236 \\
\hline AVP (pg/mL) & $65.57 \pm 33.38$ & $37.59 \pm 12.53$ & $\mathbf{P}<0.00$ I $^{*}$ \\
\hline OXT (pg/mL) & $12.95 \pm 4.84$ & $35.4 I \pm 12.75$ & $\mathbf{P}<0.00$ I $^{* *}$ \\
\hline BMI $\left(\mathrm{kg} / \mathrm{m}^{2}\right)$ & $26.22 \pm 5.79$ & $26.78 \pm 4.11$ & 0.691 \\
\hline
\end{tabular}

Note: *Significantly higher than control, **significantly lower than control. Statistically significant values are shown in bold.

Abbreviations: ANP, atrial natriuretic peptide; AVP, arginine-vasopressin; BMI, body mass index; OXT, oxytocin.

acute schizophrenia. Briefly, OXT is low in patients with acute schizophrenia, and there is a negative correlation between OXT and PANSS positivity and the CGI score, which indicates the symptom severity. On the other hand, there was a positive correlation between OXT and functionality according to the GAF scale. Conversely, the other posterior pituitary neurohormone AVP was significantly higher in schizophrenia, and there was a negative correlation between AVP and CGI score. ANP concentration was lower in schizophrenia patients with acute attack, but not in a significant level, and also it was not correlated with clinical features.

OXT, with effects ranging from maternal attachment to fear extinction, has attracted increasing attention of the researchers for the diagnosis and treatment of the schizophrenia. As in our results, a recent study supported that decreased serum levels in patients having schizophrenia correlate with neuroendocrine dysfunction and negative symptoms. ${ }^{18}$ However, there are conflicting findings about oxytocin and symptom severity. ${ }^{19,20}$ Our results suggest that OXT has a relationship with functionality of the patient and the severity of the disease as well as the positive symptoms. Functionality is an important result, and thus it affects the whole of the patients' and their relatives' life burden. ${ }^{21}$ Recently, it has been shown that nasal administration of OXT as a treatment approach has ameliorated social cognition and facial emotion recognition in schizophrenia. ${ }^{22}$ The results of our study are in line with previous studies, and it is promising for future studies about the relationship between functionality of patients having schizophrenia and the OXT.

AVP has an important role in the maintenance of water and electrolyte balance. Along with the increase in AVP, osmoregulation is disturbed and hyponatremia occurs. Hyponatremia causes changes in the mental status, eg, delirium. AVP and psychosis has previously shown to have a suspicious relationship; although psychosis, polydipsia, and appropriate AVP release constitute a clinical triad, their relationship is not clear. ${ }^{23,24}$ According to the literature, AVP concentration is increased in schizophrenia, and this result confirmed the high concentrations of AVP levels in our study. AVP was correlated with the clinical severity of schizophrenia. Previously, higher AVP levels were shown to be associated with worse anhedonia and antisocial behaviors, positive and negative symptom severity and worse cognition. ${ }^{25,26} \mathrm{We}$ could not find a relationship between AVP changes and the symptoms of schizophrenia; this is considered to be because of the sample size or the study design and since all of the cases are male in our study. According to the literature regarding the emotional behavior function, there are different findings showing the gender-dependent changes of this hormone. ${ }^{26,27}$

OXT and AVP are closely related neurohormones modulating stress responses, ${ }^{28,29}$ although their general actions often oppose to each other. ${ }^{30}$ This yin-yang type relationship

Table 3 Correlation analysis of hormonal levels and clinical features in patients with acute schizophrenia

\begin{tabular}{|c|c|c|c|c|c|c|}
\hline \multirow{2}{*}{$\begin{array}{l}\text { General characteristics and } \\
\text { clinical features of patients }\end{array}$} & \multicolumn{2}{|l|}{ OXT } & \multicolumn{2}{|l|}{ ANP } & \multicolumn{2}{|l|}{ AVP } \\
\hline & $r$ value & $P$-value & $r$ value & $P$-value & $r$ value & $P$-value \\
\hline Age & -0.057 & 0.673 & 0.183 & 0.169 & -0.023 & 0.864 \\
\hline BMI & 0.060 & 0.658 & 0.115 & 0.395 & 0.015 & 0.911 \\
\hline Duration of disease & -0.087 & 0.623 & 0.279 & 0.110 & -0.042 & 0.815 \\
\hline Onset age & 0.227 & 0.228 & -0.062 & 0.745 & 0.139 & 0.463 \\
\hline Number of hospitalizations & 0.125 & 0.480 & 0.059 & 0.739 & 0.047 & 0.792 \\
\hline Total duration of hospitalizations & 0.151 & 0.550 & -0.190 & 0.450 & 0.087 & 0.733 \\
\hline PANSS positive score & -0.373 & $P=0.030$ & -0.162 & 0.359 & -0.152 & 0.392 \\
\hline PANSS negative score & -0.168 & 0.343 & -0.134 & 0.450 & -0.105 & 0.556 \\
\hline PANSS general score & -0.224 & 0.202 & -0.085 & 0.631 & -0.004 & 0.981 \\
\hline Total PANSS score & -0.309 & 0.075 & -0.173 & 0.327 & -0.085 & 0.633 \\
\hline GAF score & 0.423 & $P=0.013$ & 0.252 & 0.151 & 0.253 & 0.149 \\
\hline CGI (severity) score & -0.465 & $P=0.006$ & -0.302 & 0.083 & -0.365 & $P=0.034$ \\
\hline
\end{tabular}

Note: Statistically significant values are shown in bold.

Abbreviations: ANP, atrial natriuretic peptide; AVP, arginine-vasopressin; BMI, body mass index; CGI, Clinical Global Impression; GAF, Global Assessment of Functionality; OXT, oxytocin; PANSS, Positive and Negative Syndrome Scale. 


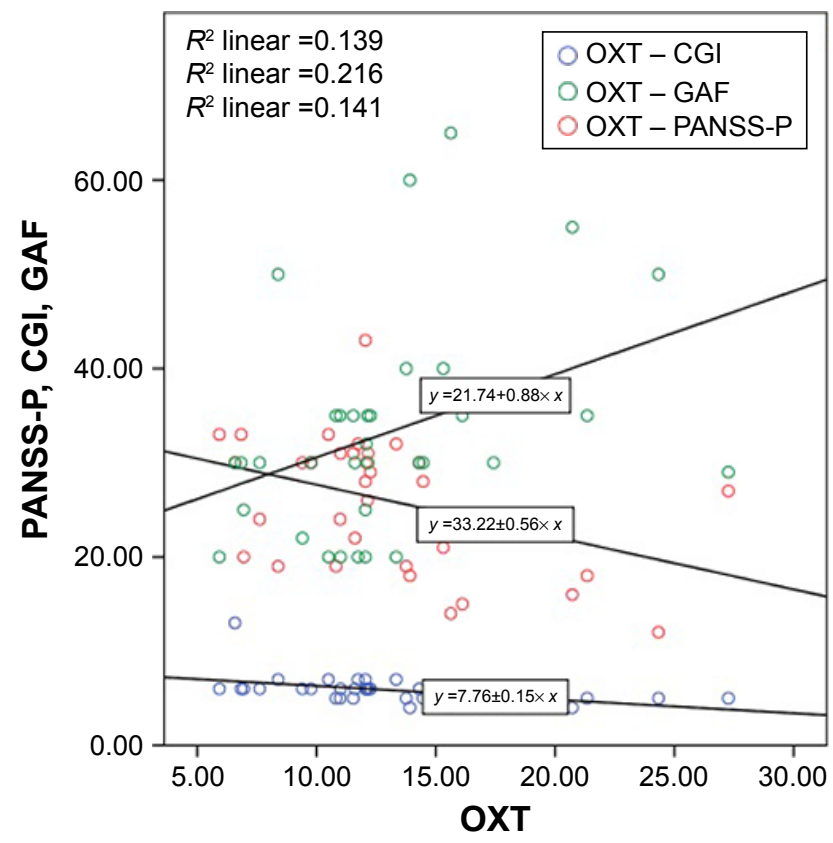

Figure I Correlation plot for OXT and PANSS-P, CGI, and GAF.

Abbreviations: CGI, Clinical Global Impression Scale; GAF, Global Assessment of Functionality; OXT, oxytocin; PANSS-P, Positive and Negative Syndrome ScalePositive symptoms.

also supports our findings and explains why AVP increases while OXT decreases in patients with acute schizophrenia.

ANP, which is secreted in response to the atrial stretch, was demonstrated to have a role in mental disorders, especially after polydipsic schizophrenia cases in 1990s, and this is in line with our findings, indicating that there is a negative correlation between ANP and AVP. ${ }^{31}$ Also, a functional relationship, and even coexistence, has been demonstrated between OXT and ANP in the literature. ${ }^{32}$ The cardiac ANP release in the bloodstream occurs after being stimulated by hypothalamic OXT according to an experimental study, whose results indicate that OXT receptors mediate the actions of OXT in releasing ANP. This, by releasing cyclic GMP, slows down the heartbeats and reduces the heart's force of contraction, in order to produce a rapid reduction in the blood volume that circulates in the body. ${ }^{33}$ These results confirm the hormonal changes that are detected in our study.

The limitation of our study is having a small sample size. Having a male sample group is both a strength and a limitation. Hormones related to gender, which have been shown to have important effects on other neurohormones, have been a serious confounding factor in previous studies. ${ }^{34}$ We eliminated this confounding factor by selecting all of the cases among the male patients. However, the changes in female patients cannot be seen in this design.
This study compares a clinical sample with healthy controls, so that the control group does not have comparable clinical features to provide variance analysis or any other correction analysis. Additionally, the patients did not have a controlled electrolyte/water intake program, which may change AVP and ANP levels. ${ }^{35}$ The sample of this study includes patients without any experimental intervention, the patients were naturally treated and observed in a psychiatry clinic so the results demonstrate the levels of OXT, AVP and ANP in natural conditions of these patients, but this study design may lead the confounding factors to be overlooked. Planning a controlled study on a larger scale and with a diet program to preserve electrolyte and fluid balance may return stronger results. The strength of our study is that this is the first acute schizophrenia study that evaluates OXT, AVP, and ANP neurohormones together and even evaluated the changes with respect to clinical features.

\section{Conclusion}

With this study, we evaluated the OXT, AVP, and ANP neurohormones together and evaluated their changes with the clinical features of acute schizophrenia. The obtained data indicate that the AVP level was higher; however, OXT and ANP levels were lower in patients having acute schizophrenia. Specifically, OXT level is related with reduced disease severity and increased functionality.

\section{Acknowledgment}

This work is supported with the funding from the Sakarya University Scientific Research and Projects Unit.

\section{Disclosure}

The authors report no conflicts of interest in this work.

\section{References}

1. Pinkham AE, Penn DL, Perkins DO, Lieberman J. Implications for the neural basis of social cognition for the study of schizophrenia. Am J Psychiatry. 2003;160(5):815-824.

2. Tsuang MT, Tohen M, Jones P. Textbook of Psychiatric Epidemiology. Hoboken, NJ: John Wiley \& Sons; 2011.

3. Kaplan HI, Sadock BJ. Synopsis of Psychiatry: Behavioral Sciences Clinical Psychiatry. Baltimore, MD: Williams \& Wilkins Co; 1988.

4. Kety SS, Rosenthal D, Wender PH, Schulsinger F. Mental illness in the biological and adoptive families of adopted schizophrenics. Am J Psychiatry. 1971;128(3):302-306.

5. Monji A, Kato T, Kanba S. Cytokines and schizophrenia: microglia hypothesis of schizophrenia. Psychiatry Clin Neurosci. 2009;63(3): 257-265.

6. Raison CL, Miller AH. When not enough is too much: the role of insufficient glucocorticoid signaling in the pathophysiology of stress-related disorders. Am J Psychiatry. 2003;160(9):1554-1565.

7. Hollander E, Bartz J, Chaplin W, et al. Oxytocin increases retention of social cognition in autism. Biol Psychiatry. 2007;61(4):498-503. 
8. Goldman MB, Gomes AM, Carter C, Lee R. Divergent effects of two different doses of intranasal oxytocin on facial affect discrimination in schizophrenic patients with and without polydipsia. Psychopharmacology. 2011;216(1):101-110.

9. Heinrichs M, Baumgartner T, Kirschbaum C, Ehlert U. Social support and oxytocin interact to suppress cortisol and subjective responses to psychosocial stress. Biol Psychiatry. 2003;54(12):1389-1398.

10. Engelmann M, Wotjak CT, Neumann I, Ludwig M, Landgraf R. Behavioral consequences of intracerebral vasopressin and oxytocin: focus on learning and memory. Neurosci Biobehav Rev. 1996;20(3):341-358.

11. Aguilera G, Pham Q, Rabadan-Diehl C. Regulation of pituitary vasopressin receptors during chronic stress: relationship to corticotroph responsiveness. J Neuroendocrinol. 1994;6(3):299-304.

12. Rinne A, Vuolteenaho O, Järvinen M, Dorn A, Arjamaa O. Atrial natriuretic polypeptides in the specific atrial granules of the rat heart: immunohistochemical and immunoelectron microscopical localization and radioimmunological quantification. Acta Histochem. 1986;80(1):19-28.

13. Chriguer RS, Rocha MJA, Antunes-Rodrigues J, Franci CR. Hypothalamic atrial natriuretic peptide and secretion of oxytocin. Brain Res. 2001;889(1-2):239-242.

14. Kay SR, Fiszbein A, Opfer LA. The positive and negative syndrome scale (PANSS) for schizophrenia. Schizophr Bull. 1987;13(2):261-276.

15. Kostakoğlu AE, Batur S, Tiryaki A, Gögüş A. Pozitif ve Negatif Sendrom Ölçeğinin (PANSS) Türkçe uyarlamasının geçerlilik ve güvenilirliği [The validity and reliability of the Positive and Negative Syndrome Scale (PANSS) for Turkish version]. Türk Psikoloji Dergisi. 1999;14(44):23-32. Turkish.

16. Guy W. EC-DEU Assessment Manual for Psychopharmacology, Revised CGI Clinical Global Impressions. Rockville, MD: US Department of Health, Education, and Welfare; 1976:76-339.

17. American Psychiatric Association. Diagnostic and Statistical Manual of Mental Disorders. 5th ed. Washington DC: American Psychiatric Association; 2013.

18. Jobst A, Dehning S, Ruf S, et al. Oxytocin and vasopressin levels are decreased in the plasma of male schizophrenia patients. Acta Neuropsychiatr. 2014;26(6):347-355.

19. Churchland PS, Winkielman P. Modulating social behavior with oxytocin: how does it work? What does it mean? Horm Behav. 2012;61(3):392-399.

20. Rubin LH, Carter CS, Drogos L, Pournajafi-Nazarloo H, Sweeney JA, Maki PM. Peripheral oxytocin is associated with reduced symptom severity in schizophrenia. Schizophr Res. 2010;124(1):13-21.

21. Yazici E, Karabulut U, Yildiz M, et al. Burden on caregivers of patients with schizophrenia and related factors. Noro Psikiyatr Ars. 2016;53(2): 96-101.

22. Averbeck B, Bobin T, Evans S, Shergill S. Emotion recognition and oxytocin in patients with schizophrenia. Psychol Med. 2012;42(2):259-266.
23. Goldman MB, Luchins DJ, Robertson GL. Mechanisms of altered water metabolism in psychotic patients with polydipsia and hyponatremia. N Engl J Med. 1988;318(7):397-403.

24. Vieweg W, Carey R, Godleski L, Tisdelle D, Pruzinsky T, Yank G. The syndrome of psychosis, intermittent hyponatremia, and polydipsia: evidence for diurnal volume expansion. Psychiatr Med. 1990; 8(4):135-144

25. Rubin LH, Demyanovich H, Wehring H, et al. M27. Peripheral oxytocin and vasopressin are associated with clinical symptom severity and cognitive functioning in midlife women with chronic schizophrenia. Schizophr Bull. 2017;43(Suppl 1):S221.

26. Rubin LH, Carter CS, Bishop JR, et al. Peripheral vasopressin but not oxytocin relates to severity of acute psychosis in women with acutely-ill untreated first-episode psychosis. Schizophr Res. 2013; 146(1-3):138-143.

27. Thompson RR, George K, Walton JC, Orr SP, Benson J. Sex-specific influences of vasopressin on human social communication. Proc Natl Acad Sci U S A. 2006;103(20):7889-7894.

28. Carter CS, Pournajafi-Nazarloo H, Kramer KM, et al. Oxytocin: behavioral associations and potential as a salivary biomarker. Ann N Y Acad Sci. 2007;1098(1):312-322.

29. Parker KJ, Buckmaster CL, Schatzberg AF, Lyons DM. Intranasal oxytocin administration attenuates the ACTH stress response in monkeys. Psychoneuroendocrinology. 2005;30(9):924-929.

30. Legros JJ. Inhibitory effect of oxytocin on corticotrope function in humans: are vasopressin and oxytocin ying-yang neurohormones? Psychoneuroendocrinology. 2001;26(7):649-655.

31. Ohsawa H, Kishimoto T, Shimayoshi N, et al. Atrial natriuretic peptide and arginine vasopressin secretion in schizophrenic patients. Acta Psychiatr Scand. 1993;88(2):130-134.

32. Jirikowski G, Back H, Forssmann W, Stumpf W. Coexistence of atrial natriuretic factor (ANF) and oxytocin in neurons of the rat hypothalamus. Neuropeptides. 1986;8(3):243-249.

33. Gutkowska J, Jankowski M, Lambert C, Mukaddam-Daher S, Zingg HH, McCann SM. Oxytocin releases atrial natriuretic peptide by combining with oxytocin receptors in the heart. Proc Natl Acad Sci U S A. 1997;94(21):11704-11709.

34. Rubin LH, Connelly JJ, Reilly JL, et al. Sex and diagnosis-specific associations between DNA methylation of the oxytocin receptor gene with emotion processing and temporal-limbic and prefrontal brain volumes in psychotic disorders. Biol Psychiatry Cogn Neurosci Neuroimaging. 2016;1(2):141-151.

35. Sailer C, Winzeler B, Christ-Crain M. Primary polydipsia in the medical and psychiatric patient: characteristics, complications and therapy. Swiss Med Wkly. 2017;147:w14514-w14514.
Neuropsychiatric Disease and Treatment

\section{Publish your work in this journal}

Neuropsychiatric Disease and Treatment is an international, peerreviewed journal of clinical therapeutics and pharmacology focusing on concise rapid reporting of clinical or pre-clinical studies on a range of neuropsychiatric and neurological disorders. This journal is indexed on PubMed Central, the 'PsycINFO' database and CAS,
Dovepress

and is the official journal of The International Neuropsychiatric Association (INA). The manuscript management system is completely online and includes a very quick and fair peer-review system, which is all easy to use. Visit http://www.dovepress.com/testimonials.php to read real quotes from published authors. 